Editorial:

\title{
VACCINE RACE FOR COVID-19 BATTLE
}

\section{By Dr. Dharma Prasad Khanal}

Till the day (8/22/2021) that I am writing this editorial World Health Organization's (WHO) dash board shows that there are 46,369,454 COVID -19 confirmed cases and 746, 833 confirm Covid-19 deaths in the world. According to WHO "Scientists around the world are working faster than ever to develop and produce vaccines that can stop the spread of COVID-19. Since the emergence of this novel corona virus in December 2019, 20 vaccines have started to be rolled out in countries worldwide. Around the world, there are now 112 COVID-19 vaccine candidates undergoing clinical trials and 183 candidates in pre-clinical development."

There are four types of COVID-19 vaccines: 1) Whole virus, 2) Spike protein subunit, 3) Nucleic acids (RNA \& DNA) and 4) Viral vector. In the vaccine production, there are two main approaches. A disease-causing COVID -19 viruses that is weakened in a laboratory so it cannot cause disease (live attenuated vaccines) that trigger protective immunity to it. Another is whole virus vaccines in deactivated form of the COVID -19 viruses that causes a disease to trigger protective immunity to it. In Inactivated vaccines viruses whose genetic material has been destroyed so they cannot replicate. Both live attenuated and inactivated vaccines contain the whole or part of the disease-causing pathogen, but the type of immunity they trigger is slightly different. According to WHO "Once large amounts of virus or bacteria have been grown in culture media, they must then be isolated, purified and attenuated or inactivated, depending on the vaccine. Each of these steps requires specific equipment, reagents, and stringent procedures to avoid, and check for, contamination, which can further increase costs".

Spike protein-based vaccines, which use harmless fragments of proteins or protein shells that mimic the COVID-19 virus to safely generate an immune response. RNA and DNA vaccines, a cutting-edge approach that uses genetically engineered RNA or DNA to generate a protein that itself safely prompts an immune response. Viral vector vaccines, which use a safe virus that cannot cause disease but serves as a platform to produce corona virus proteins to generate an immune response.Presently the vaccine race is as following:

$\begin{array}{lllllll}\text { Pre- clinical } & \text { Phase I } & \text { Phase II } & \text { Phase III } & \text { In use } & \text { Phase IV } & \text { Source } \\ 184 & 37 & 37 & 28 & 20 & 8 & \text { WHO (4) }\end{array}$


There is a common consciences among the researchers that it's too early to know the duration of protection of COVID-19 vaccines. Research is ongoing to answer this question. However, it's encouraging that available data suggest that most people who recover from COVID-19 develop an immune response that provides at least some period of protection against reinjection although researchers are still learning how strong this protection is, and how long it lasts. There are several variants of COVID-19 virus. According to WHO "most vaccines stimulate enough immunity to retain substantial efficacy against most variants, especially for severe disease, hospitalization, and death" WHO designated the variant B.1.1.529 a variant of concern, named Omicron". It is not yet clear whether Omicron is more transmissible (e.g., more easily spread from person to person) compared to other variants, including Delta and presently used variant of vaccines are effective against it or not? So WHO is working with technical partners to understand the potential impact of this variant on our existing countermeasures, including vaccines.

1. Tauseef Ahmad, Manal Abdulaziz Murad, Mukhtiar Baig \& Jin Hui (2021) Research trends in COVID-19 vaccine: a bibliometric analysis, Human Vaccines \& Immunotherapeutics, DOI: 10.1080/21645515.2021.1886806

2. https://www.who.int/news-room/q-a-detail/coronavirus-disease-(covid-19)-vaccines?

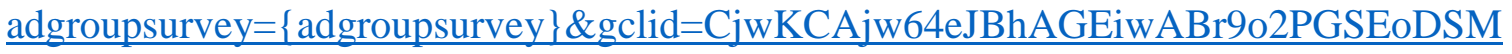
Nz0md3Qkh6ntfTmGZBOGhy7dusaU04_43WNz0s4KAKN5hoCf2gQAvD_BwE\#/ Retrieve on $8 / 22 / 2021$.

3. https://www.gavi.org/vaccineswork/covid-19-vaccine-race. Retrieve on 8/22/2021.

4. https://www.who.int/publications/m/item/draft-landscape-of-covid-19-candidatevaccines. Retrieve on 8/22/2021.

5. Saman Soleimanpour \& Atieh Yaghoubi (2021) COVID-19 vaccine: where are we now and where should we go?, Expert Review of Vaccines, 20:1, 23-44, DOI: $\underline{10.1080 /}$ 14760584. 2021.1875824.

6. Tushar Yadav, Nishant Srivastava, Gourav Mishra, Kuldeep Dhama, Swatantra Kumar, Bipin Puri \& Shailendra K Saxena (2020) Recombinant vaccines for COVID-19, Human Vaccines \& Immunotherapeutics, 16:12, 2905-2912, DOI: $\underline{10.1080 / 21645515 \text {.2020. }}$ $\underline{1820808 .}$.

7. https://www.who.int/news/item/28-11-2021-update-on-omicron. Retrieve on 12/2/2021. 